\title{
Investigations on Issues in Microgrids
}

\author{
Prerna Gaur and Sunita Singh
}

\begin{abstract}
This paper presents many technical issues and shortcomings confronted in installation of micro-grids particularly in rural areas. It discusses the technical challenges involved in grid integration of micro-grids and highlights some aspects of stability in micro-grids. There are different types of micro-grid applications. The system layout and the control design vary depending on the application and so does the facet of stability in a micro-grid. It briefly encompasses the stability aspects of remote, utility connected micro-grids depending on the modes of operation, control topology, types of renewables and the network parameters. In the end, a brief insight into IEC 62257 specifications has been given.
\end{abstract}

Index Terms-Power quality, smart grid, solar power, stability, wind power.

\section{INTRODUCTION}

Microgrids are small power systems capable of island and grid modes of operation. Managing their power balance and stability maybe a difficult task since they depend on quite a number of variables. A microgrid is a controllable component of the smart grid defined as a part of distribution network capable of supplying its own local load even in the case of disconnection from the upstream network. For reasons of reliability, distributed generation resources would be interconnected to the same transmission grid as central stations. They use renewable energy sources, including, but not limited to wind energy, biomass, biogas, solar energy, fuel cells etc. Various technical and economic issues occur in the integration of these resources into a grid. Technical problems arise in the areas of power quality, voltage stability, harmonics, reliability, protection, and control. Behavior of protective devices on the grid must be examined for all combinations of distributed and central station generation. A large scale deployment of distributed generation may affect grid-wide functions such as frequency control and allocation of reserves. A microgrid (MG) is a localized grouping of electricity generation, energy storage, and loads that normally operates connected to a traditional centralized grid (macrogrid). This single point of common coupling with the macrogrid can be disconnected. The microgrid can then function autonomously [1], [2]. Maintaining a near sinusoidal power distribution bus voltage at rated magnitude and frequency is referred to as electric power quality. Voltage unbalance, harmonic content, increased reactive power demand, and frequency deviation are the foremost power quality hitches which affect the utility grid power quality,

Manuscript received March 20, 2015; revised December 1, 2015.

Prerna Gaur is with Netaji Subhas Institute of Technology, Delhi, India (e-mail: prernagaur@yahoo.com).

Sunita Singh is with the ABES Institute of Technology, Ghaziabad, India (e-mail: singh9sunita@ rediffmail.com). voltage stability, harmonics, reliability, protection, and control [3]. Power electronics devices have to be incorporated in order to maintain the balance between load and generated power, and to guarantee the quality of supply on an acceptable level. Strategies have to be developed in order to improve voltage sags/swells and unbalances. Static synchronous compensators are widely used for this purpose [4]. Enhanced networked-based control structure is needed not only to eliminate the frequency deviations, power-sharing errors, and stability concerns associated with conventional droop control in microgrids but also to yield improved microgrid dynamic performance, minimized active/reactive power-sharing errors under unknown line impedances, and high reliability and robustness against network failures or communication delays.

\section{ISSUES}

\section{A. Power Imbalance}

A power imbalance occurs in the transition from grid-connected to islanded mode if the microgrid is absorbing or supplying power to the main grid before disconnection. Energy storage units are used to maintain power balance due to the slow dynamic response and low inertia of some micro sources. When a microgrid once again goes from islanded mode to grid connected mode, it is synchronised by ensuring the magnitude and phase of the voltages across the synchronising device are equal. A high-speed static switch with appropriate sensing capability may be used for disconnection [5].

\section{B. Stability Issues in Smart Grids}

The stability issues in smart grids with high penetration of DGs and MGs arising from their diverse operational characteristics compared to conventional plants are depicted in this section.

1) Lower Angular Stability due to lower overall system inertia: In standard powergrids, the inertial support from the rotating mass of the synchronous generators plays a significant role for maintaining the grid frequency during transient periods. In smart grids, greater part of the DGs and MGs are integrated to the main grid through power-electronic based converters.

These power electronic converters electrically decouple the DGs and MGs from the smart grid when failures take place in the smart grid. Hence, these DGs and MGs normally render no inertial backing during power grid disturbances, such as transmission line faults, unforeseen load change, etc. Hence, the increased infiltration of DGs and MGs results in lower system inertia for the smart grid. The reduced the system inertia is, the bigger the susceptibility is for smart grid to voltage and frequency instabilities due to transients. 
2) Lower Voltage Stability due to lower power distribution support: Since the power electronic converters in DGs and MGs are operated in the current control mode, the power transshipped to the smart grid is limited to preset figures. Therefore, during crisis in the smart grid, the power sharing support from DGs is much less compared to that of regular power plants. Traditional power plants can meet the increased power requirements during various eventualities with the kinetic energy stored in the rotating mass of synchronous generators. As MGs are switched to autonomous mode of functioning during contingencies in the main power grid, the power sharing support from MGs can be considered as zero. Hence, the current controlled functioning of DGs and islanded operation of MGs can deteriorate the total power generation within the smart grid, in turn rejecting the voltage profile. Therefore, high penetration of DGs and MGs can deteriorate the immunity of the voltage profile of the smart grid during various power system outages.

3) Low-frequency power oscillations: The MPPT algorithm in the grid side controller (GSC) of a DG sets the maximum power output value for the DG inverter for that operating condition of renewable source. Depending on the frequency of the MPPT refurbished, the output of the DG inverter will have deviations. These deviations in each DG in the smart grid can sum up to give rise to oscillations in total power generation. These ceaseless changes in the power sharing ratio of DGs can produce rotor oscillations in synchronous generators. These oscillations can be serious when the inception of DGs becomes very high. The influence of these power oscillations on the operation of MGs is of major cause for worry, as MGs are designed to remodel to islanded mode during disturbances in the main grid. The controller at the MG may identify these power oscillations as a disturbance and may drive the MG to islanded mode. This will amplify the power oscillations, leading to aggravation of the transient state of the smart grid. If the rotor oscillations in regular power plants are left unnoticed, it may lead to serious power fluctuations in the smart grid and can lead to catastrophic failures. Hence, power transients in the smart grid have to be analyzed and damped in real time [6].

\section{Harmonics in DC Microgrids}

The Harmonic currents need to be reduced to reduce harmonic losses. For this harmonic compensation methods have to been proposed as in [7]. The harmonics result due to operation of power electronic converters. The harmonic voltage and current should be limited to the acceptable level at the point of wind turbine connection to the network. To ensure that harmonic voltage is within acceptable values, each source of harmonic current can allow only a limited contribution, as per the IEC-61400-36 guideline. The brisk switching amounts to large contraction in lower order harmonic currents compared to the line commutated converter, but the output current will have increased frequency current and can be comfortably filtered-out.

\section{Controllers for Regulation of Power Flow}

Inspired by control architectures from transmission level power systems, regulation in microgrids is generally approached in a hierarchical manner. The first and most basic level is primary control, which is related with the stability of and load sharing within the electrical network. While generally successful, these decentralized "droop" controllers typically force both the local voltages and the steady-state network frequency to deviate from their nominal values. Then is the secondary control level and finally the tertiary level. In [8] a distributed controller is designed for regulating power flow among multiple microgrids in islanded modes with variable renewable energy sources (RESs) while minimizing dynamic interactions. The controller is devised in a manner that it has the capability of gathering information from the neighboring microgrids. Both modal analysis and nonlinear simulations are used to identify the critical issues that might degrade the stability under different operating conditions. The proposed control scheme is validated on a test distribution system with multiple microgrids and time-domain simulations are performed to verify its effectiveness of the control scheme. It is found that the controller implemented provides excellent performance in minimizing the negative interactions through the regulation of power flow among multiple microgrids in the islanded mode.

\section{E. Inverters for DC-AC Conversion}

Microgrids are local low voltage power systems that offer many advantages over grid power in terms of dependability, power discharge and the ability to incorporate distributed generation, including renewable energy. In low voltage AC microgrids, especially where solar arrays are used, inverters are required to convert $\mathrm{DC}$ to $\mathrm{AC}$. With a large number of inverters in a microgrid, there are concerns regarding their ability to support such a small power system when it is under large stress. Such stress can be caused by voltage and frequency deviations arising inside or outside the microgrid, or from faulty situations. Currently, microgrids rely upon accurate design while being manufactured for them to operate steadily. Inverters available in the market offer no more than power conversion at unity power factor. However, the newly planned microgrids are likely to be better structured, and inverters will have the capacity to do something more. They may play a role in regulating flow of reactive power and in reducing harmonics, or may offer supplementary services [9].

\section{F. Frequency and Active Power Control}

The transition to islanded operation mode and the operation of the network in islanded mode require micro generation sources to particulate in active power frequency control, so that the generation can match the load. During this transient period, the participation of the storage devices in system operation is indeed very vital, since the system has very low inertia, and some micro sources (micro turbine and fuel cell) have very slow response to the power generation increase. As already stated, the power necessary to provide appropriate load following is obtained from storage devices. Knowing the network characteristics, it is possible to define the maximum frequency droop as shown in Fig. 1. To maintain the frequency between acceptable limits, the $\mathrm{V} / \mathrm{f}$ inverter connected to storage device will adjust the active power in the network. It will inject active power when frequency falls from the nominal value and will absorb active 
power if the frequency rises above its nominal value [10].

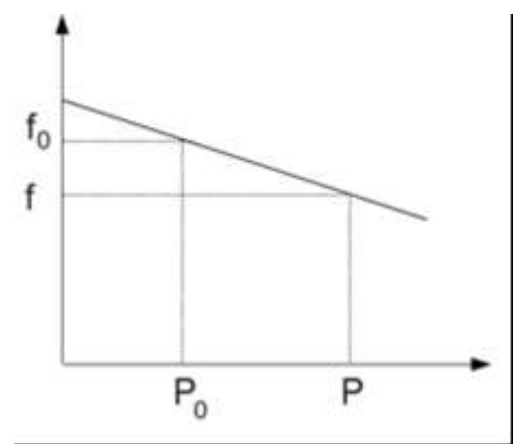

Fig. 1. Frequency droop characteristic.

\section{G. Voltage and Reactive Power Control}

Variation in amplitude of supply voltage could be relieved by adopting reactive power compensation technology due to voltage reactive power droop characteristic. Fig. 2 shows a general voltage droop characteristics. By knowing the network characteristics, it is possible to define the maximum voltage droop. In [11], a voltage sources inverter (VSI) or V/f inverter is connected to the flywheel to maintain the voltage between acceptable limits. It will adjust the reactive power in the microgrid. It will inject reactive power when voltage falls from the nominal value and will absorb reactive power if the voltage rises above its nominal value.

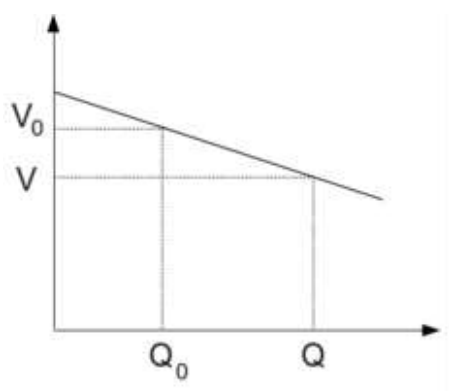

Fig. 2. Voltage droop characteristic.

\section{H. Topological Changes}

Microgrids operate in two modes: grid connected and stand-alone. Topological changes take place in low voltage networks due to connection/disconnection of generators, storage systems and loads. Also, there is intermittence in the generation of several renewable resources connected in the microgrid. Inception of information and communication technologies to the power grid technologies is changing the architecture and operation of the regular grid. Micro grid topology can be installed in areas such as city buildings, factories, households, villages or rural farms. For different cases, depending on user requirements, strength of the utility grid, and the number of available distributed energy resources (DER), ac, dc or hybrid microgrid can be put up. To improve the dependability of the microgrid, an energy storage system is established to support the bus voltage when the microgrid goes from the grid connected mode to autonomous mode. This energy storage system can be charged from the main bus voltage through a converter. Fig. 3 shows the architecture of a typical microgrid. Microgrids, overall, are controlled by the microgrid control center [12].

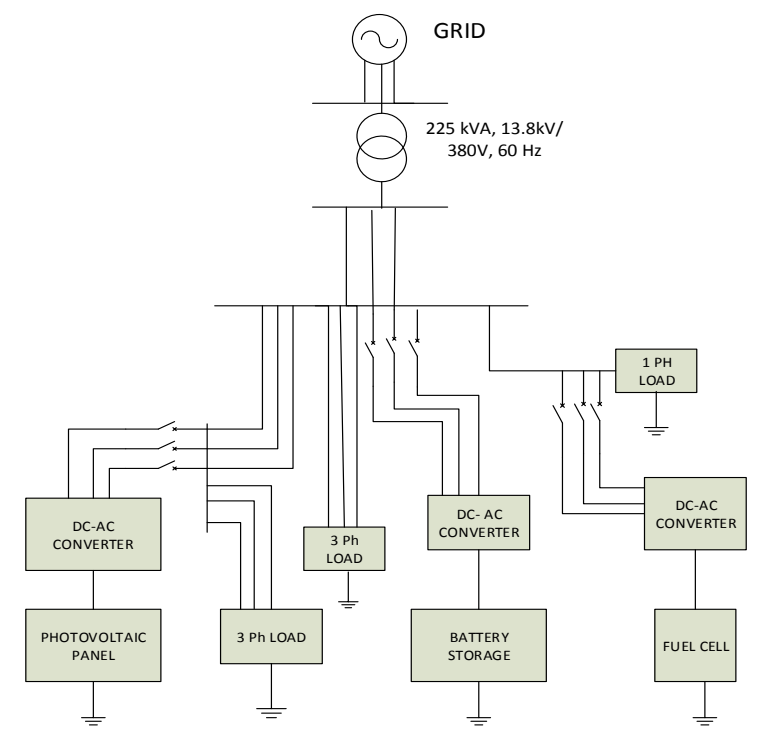

Fig. 3. Architecture of a typical microgrid.

\section{Protection Issues}

An effective dawn of distributed generation (DG) into existing distribution networks (DNs) calls for a replacement of traditional power system protection concepts and strategies [13]. New issues such as activeness of DNs and bidirectional power flow require new protection plans. Behavior of protective devices on the grid must be examined for all combinations of distributed and central station generation. Distribution feeder protection could soon be elaborate by non radial flows of real and reactive power available from high penetration distribute generation and more specifically from microgrids. Non directional overcurrent safeguard may not provide necessary security and sensitivity for faults on remote points of the network. Directional surveillance is necessary to set overcurrent pickups with adequate emphasis on remote faults. Mounting the directional element by classical means provides a reliability risk at varying VAR flows within reach of specific types of renewable energy generation. A unique solution using directional overcurrent elements and supplemented by a load encroachment function can solve these problems. In [14] this approach has been substantiated in renewable plant collector circuit protection applications over a wide range of operating conditions.

\section{J. Energy Storage Devices}

As diesel generation and renewables are intermittent means of electricity production, fluctuations resulting from unstable micro-sources and nonlinear loads will execute considerable impacts on normal operation of the MG. Energy storage technology puts forward a preferable solution to the above concern. Energy storage is used to enhance the stability and efficiency of microgrids by disconnecting the generation source from the load. By providing power and lighting during large-scale catastrophical conditions such as rain and storm , energy storage systems of all types reduce the time it takes for first responders to begin recovery actions. Energy storage mechanisms and topologies are being developed and the features and benefits of energy storage systems are being reviewed. One such example of an upcoming energy storage technology is the recent introduction of sodium-nickel-based batteries to the marketplace. Other storage methods include: 
lead acid battery, rechargeable battery, flow battery, super-capacitor, superconducting magnetic energy storage, hydroelectric or pumped energy storage, flywheel energy storage, compressed air energy storage, thermal energy storage, molten salt, ice storage [15].

\section{K. Economic Aspects}

Concepts of microgrids are proposed to address primarily various issues related to integration of small scale renewables and increased demand of reliable electricity supply. With an active management control approach and ability to operate in islanding mode, a cluster of micro generators, electricity storage and electrical loads can be operated within the microgrids framework to provide higher supply reliability to customers. Solutions are required not only to make these concepts technologically feasible and safe to operate but also to be commercially viable and attractive, economically efficient and supported by electricity regulations. Detailed study of investigations on various economic, regulatory and commercial issues faced by the development of microgrids in projects is need of the hour. The potential economic benefits and contributions to environment from applications of microgrid technologies should be taken into consideration [16].

\section{IEC 62257 SPECIFICATIONS}

The IEC 62257 series introduces general considerations on rural electrification. This series intends to provide to different players involved in rural electrification projects specifications for the setting up of low voltage renewable energy and hybrid systems. International standards can be used to safeguard quality and sustainability. IEC Technical Specification Series 62257 is a comprehensive set of standards covering the technical and organizational aspects of mini-grids. These standards [17] provide a comprehensive and logical framework for the design, installation, and maintenance of mini-grids, with a particular emphasis on safety, user friendliness and efficiency. Moreover, the standards give an overview on important contractual questions and technical implementation plans (Series 3 project development and management). Business plans, financing schemes, and the range of social challenges (embedding into the community) are not covered by this series.

Since the IEC series 62257 can be seen as a series of checklists, it is possible to envisage the self-declaration of planners and installers that they have complied with the approach suggested by this series. This would require comprehensive reporting which gives answers to all relevant aspects suggested by the series. In this sense, it could serve as a solid technical quality standard. The IEC series was published from 2003 to 2008. Since this series is more an approach than guidance on specific technology, it is not likely to be outdated quickly, even as technologies evolve.

\section{CONCLUSION}

Hybrid energy systems is a comparatively recent concept and considerable research and development activities are still continuing in the area to help address existing challenges and facilitate these systems in playing a vital role in the world's future energy system. This paper presents a review of the issues in implementation of microgrid technology. It introduces the activities and current progress in microgrid research, especially in various countries of Europe and America. The three key issues of technical challenges that must be overcome for effective implementation of microgrid are voltage and frequency control, islanding and protection. In this context, integration of small-scale production in the form of microgrids, supported by the inclusion of power electronic devices, could possibly contribute largely to the improvements in power quality as seen by end users. In upcoming power generation layouts, the microgrid will provide clear economic and environmental benefits as compared to traditional power system. Efforts for development and application of concepts is required to resolve numerous economic, commercial and technical challenges. Combined efforts and exchange of information among researchers on these activities will aid in carrying the microgrid research endeavors further.

\section{NOMENCLATURE}

$f$ is the system frequency;

$f_{0}$ is the base frequency;

$K_{p}$ is the frequency droop control setting;

$P$ is the active power of the unit;

$P_{0}$ is the base active power of the unit;

$V$ is voltage at the referred location;

$V_{0}$ is the base voltage;

$Q$ is the reactive power of the system;

$Q_{0}$ is the base reactive power;

$K_{q}$ is the voltage droop control setting.

\section{REFERENCES}

[1] A. S. Dobakhshari, S. Azizi, and A. M. Ranjbar, "Control of microgrids: aspects and prospects," in Proc. IEEE Int. Conf. on Networking, Sensing and Control, Apr. 11-13, 2011, pp. 38-43.

[2] K. Kauhaniemi and O. Palizban, "Microgrid control principles in island mode operation," in Proc. IEEE Power Tech. Conf., Grenoble, France, Jun. 16-20, 2013, pp. 1-6.

[3] W. Al-Saedi, S. W. Lachowicz, D. Habibi, and O. Bass, "Power flow control in grid-connected microgrid operation using particle swarm optimization under variable load conditions," Int. Journal of Electric. Power \& Ener. Syst., vol. 49, pp. 76-85, 2013.

[4] M. Barnes and P. Korba, "Smart grids - microgrid systems," Intelligent Automation and Soft Computing, vol. 16, no. 2, pp. 195-198, 2010.

[5] Z. Xiao, J. Wu, and N. Jenkins, "An overview of microgrid control," Intelligent Automation and Soft Computing, vol. 16, no. 2, pp. 199-212, 2010.

[6] P. Gopakumara, M. J. Reddya, and D. K. Mohanta, "Stability concerns in smart grid with emerging renewable energy technologies," Electric Power Components and Sys., vol. 42, no. 3, pp. 418-425, 2014.

[7] W. Feng, M. Vilathgamuwa, and S. S. Choi, "Mitigation of harmonics of DFIGs in DC microgrids," in Proc. IEEE Energy Convers. Congress \& Expo., Singapore, Sept. 15-20, 2012, pp. 1946-1953.

[8] M. J. Hossain, M. A. Mahmud, H. R. Pota, and N. Mithulananthan, "Distributed control scheme to regulate power flow and minimize interactions in multiple microgrids," in Proc. IEEE PES Gen. Meeting Conf. \& Expo., Jul. 27-31, 2014, pp. 1-5.

[9] T. Moore and S. Sayeef, "Challenges and opportunities for inverters in microgrids," in Proc. Conf. on IEEE Industrial Electronics Society, Melbourne, Nov. 7-11, 2011, pp. 3111-3116.

[10] J. Jimeno, J. Anduaga, J. Oyarzabal, and A. G. de Muro, “Architecture of a microgrid energy management system," European Trans. on 
Electrical Power, vol. 21, no. 2, pp. 1142-1158, March 2011.

[11] K. Koyanagi , A. Taguchi, M. Ishimaru, and R. Yokoyama, "Study of the load-following performance of distributed generators in a micro-grid," Trans on Electrical and Electronics Engg., vol. 3 , no. 5, pp. 492-502, Sep. 2008.

[12] D. H. Pham, G.Hunter, L. Li, and J. Zhu, "Microgrid topology for different applications in Vietnam," in Proc. 22nd Australasian Universities Power Engineering Conference, Bali, 26-29 Sept. 2012, pp. 1-6.

[13] G. Buigues, A. Dyśko, V. Valverde, I. Zamora, and E. Fernández, "Microgrid protection: Technical challenges and existing techniques," in Proc. Int. Conf. on Renewable Energies and Power Quality ICREPQ'13, Bilbao, Spain, Mar. 20-22, 2013.

[14] D. Jones and J. J. Kumm, "Future distribution feeder protection using directional overcurrent elements," IEEE Trans. Ind. Appl., vol. 50, no. 2, pp. 1385-1390, Sep. 2013.

[15] X. Tan, Q. Li, and H. Wang, "Advances and trends of energy storage technology in microgrid," Int. Journal of Electrical Power \& Energy Syst., vol. 44, no. 1, pp. 179-191, Jan. 2013.

[16] S. Bando and H. Asano, "Economic evaluation of microgrids," in Proc PES General Meeting - Conversion and Delivery of Electrical Energy in the 21st Century, Pittsburg, Jul. 20-24, 2008, pp. 1-6.
[17] S. Rolland and G. Glania, "Hybrid mini grids for rural electrification, lessons learned," US Academy Inter. Dev., Mar. 2011.

Prerna Gaur obtained her bachelor's degree in electrical engineering from G B Pant University and master degree from Delhi University. She is presently working as an associate professor in the Department of Instrumentation and Control Engineering, Netaji Subhas Institute of Technology, New Delhi.

Sunita Singh was born in Rourkela, India, in 1968. She completed her graduation as well as post graduation in electrical engineering from National Institute of Technology, Rourkela, India in 1994. She is currently pursuing her $\mathrm{PhD}$ degree in power systems and renewables from Uttar Pradesh Technical University. Her area of research work is micro-grids and renewables.

She is presently working as an associate professor at ABES IT, Ghaziabad. Prior to this, she was the head of Department at Ideal Institute of Management and Technology. She has around 15 years of teaching experience. Prof. Singh is a member of professional societies like, IEEE, IE and ISTE. She has presented research papers in conferences in various reputed institutes including the Indian Institute of Technology, Mandi. 